\title{
Neurodevelopmental Outcome at 12 Months of Postnatal Magnesium Sulphate Therapy for Perinatal Asphyxia
}

\author{
Prakash R ${ }^{1}$, M R Savitha², B Krishnamurthy ${ }^{3}$
}

${ }^{1}$ Dr. Raj Prakash MBBS. MD, Professor, ${ }^{2} \mathrm{Dr}$. MR Savitha MBBS. MD. DNB, Professor, ${ }^{3} \mathrm{Dr}$. B Krishnamurthy MBBS. MD. DCH. All from the Department of Paediatrics, Mysore Medical College and Research Institute, Mysore, Karnataka, India.

\author{
Address for correspondence: \\ Dr. Raj Prakash, \\ Kaithathu-Revathy, \\ Peringala PO, Kayamkulam, \\ Kerala, India. \\ Phone: +919846023482 \\ E-mail: rajprakash84@gmail.com
}

\author{
Acknowledgements: None \\ Funding: Nil \\ Conflict of Interest: None \\ Permission from IRB: Yes \\ Ethical dilemmas faced during study: No
}

\begin{abstract}
How to cite
Prakash R, M R Savitha, B Krishnamurthy. Neurodevelopmental Outcome at 12 Months of Postnatal Magnesium Sulphate Therapy for Perinatal Asphyxia. J Nepal Paediatr Soc 2016;36(3):256-262.
\end{abstract}

doi: http://dx.doi.org/10.3126/jnps.v36i3.15565

This work is licensed under a Creative Commons Attribution 3.0 License.

\begin{abstract}
Introduction: Postnatal magnesium therapy has been proposed as a novel neuroprotective agent for perinatal asphyxia. A few studies reported short term neurological benefit with magnesium. It is uncertain whether magnesium therapy has any long term effect on neurodevelopment. Material and Methods: We randomly assigned 120 term asphyxiated infants to receive either magnesium sulphate infusion or placebo postnatally in first 48 hours of life. Babies were treated as per the standard treatment protocol for asphyxia. Short term outcome at discharge was previously reported and a follow up evaluation at 12 months was done. The primary outcome was a composite of death or disability, developmental delay and neuromotor tone abnormality at 12 months. Results: Out of 120 infants, 69 infants had moderate-severe hypoxic-ischemic encephalopathy (HIE) during initial NICU stay. Among 69 infants with moderate-severe HIE, 41 infant could be followed up. Out of 41 infants, 22 were in magnesium group and 19 in placebo group. Of 22 infants assigned to magnesium therapy, $3(13.6 \%)$ died or survived with neurodevelopmental disability as compared with 5 of 19 infants $(26.3 \%)$ assigned to placebo $(p=0.32)$. The developmental outcome evaluated found developmental delay in 3 of 22 infants in magnesium group vs 5 of 19 infants in placebo group $(p=0.32$ ). AmeilTisonneuromotor tone assessment revealed tone abnormality in 3 of 22 infants in study group vs 4 of 19 infants in placebo group $(p=0.53)$. Conclusion: Magnesium therapy for perinatal asphyxia may not result in favourable long term neurodevelopmental outcome, though no significant adverse effect has been documented.
\end{abstract}

Key words: Magnesium sulphate; Newborn, Neurodevelopmental outcome, Perinatal asphyxia

\section{Introduction}

erinatal asphyxia is an important cause of early neonatal death and stillbirth. World Health Organization (WHO) defines birth asphyxia as failure to initiate and sustain breathing at birth. According to latest estimates by $\mathrm{WHO}$, approximately 4 million babies die each year before they reach the age of one month. Ninetyeight percent of these neonatal deaths take place in the developing 
countries. Perinatal asphyxia and birth injuries together contribute to almost $29 \%$ of these deaths ${ }^{1}$.The clinical manifestations of asphyxia have been termed as Hypoxic Ischemic Encephalopathy (HIE). HIE is of concern in an asphyxiated neonate because it can lead to serious long-term neuro-motor sequelae among survivors ${ }^{2,3}$. As of today, the management of asphyxiated babies is mainly supportive and involves maintaining optimum temperature, oxygenation, ventilation, perfusion, metabolic milieu and control of seizures. In addition several newer modalities of therapy have been under research including therapeutic hypothermia. A large number of drugs are under investigation for neuroprotection in HIE. These need to be used in the early period of hypoxic ischemic injury. They act by causing blockade of free radical generation (allopurinol, oxypurinol), scavenging of oxidants (superoxide dismutase, glutathione, $\mathrm{N}$-acetyl cysteine and alpha tocopherol), calcium channel blockade (flunarizine, nimodipine), blockade of NMDA receptors (magnesium, MK801, dextromethorphan) and blocking inflammatory mediators (phospholipase A2, indomethcin).

In perinatal asphyxia, glutamate, which is the main excitatory aminoacid neurotransmitter, is released in large amount into brain extracellular compartment. Glutamate binds to the NMDA ( $\mathrm{N}$ - methyl D-Aspartate) receptors causing opening of calcium channels and influx of calcium into neuronal cells $s^{4,5}$. Calcium will trigger a cascade of intracellular events mediating cell death. Magnesium, as a naturally occurring NMDA receptor antagonist, has been proposed as a novel therapeutic agent to prevent glutamate excitotoxicity and brain damage $e^{6,7,8}$.

Short term beneficial effect has been reported in studies done to evaluate postnatal magnesium therapy for perinatal asphyxia. Our own previous study evaluated the short term neurological outcome, which was beneficial and favoring magnesium therapy ${ }^{9}$. The literature is sparse in terms of long term outcome.

Aim of the present study is to evaluate the neurological outcome at 12 months of infants treated with magnesium for perinatal asphyxia.

\section{Material and Methods}

The present study was conducted in term neonates with perinatal asphyxia delivered at Mother and Child hospital attached to a tertiary care teaching medical college in India from November 2011 to February 2012. A minimum sample size calculation of 120 infants (60 study group and 60 comparison group) with perinatal asphyxia was done with the incidence of perinatal asphyxia of 2/100 live births at our institution at a 0.05 significance level and a power of $80 \%$. The formula used was $n=z^{2} \mathrm{pq} / \mathrm{d}^{2}$ where $z=1.96, p=0.02, q=1-p, d=0.05$.

The infants were enrolled when following inclusion criteria were met: 1) A sentinel hypoxic event occurring immediately before or during labour. 2) Failure to initiate breath at birth or APGAR score $<7$ at one minute.3) Need for resuscitation at birth (positive pressure ventilationor chest compression) 4) Early onset features of hypoxic ischemic encephalopathy.5) Exclusion of other etiologies for encephalopathy.

Preterm infants, infants whose mothers received pethidine or phenobarbitone which might cause depression in baby and neonates with any obvious external congenital malformations were excluded from the study. The methodology and results of short term outcome at discharge have been explained in our previous study $^{9}$

As soon as the baby was admitted to NICU, the details were entered in a predesigned proforma. The infants were assigned randomly, using a computer generated random number table, to receive either magnesium sulphate infusion (study group) or normal saline (control group). The sequentially numbered allocation was not concealed. Allocation to either of group was done within first six hours of life. All neonates were treated according to the routine NICU protocol for perinatal asphyxia. The supportive management included maintenance of adequate oxygenation and ventilation, maintenance of sufficient brain and organ perfusion, normal metabolic status, maintenance of euthermic and euglycemic status, fluid and electrolyte balance and control of seizures. Therapeutic hypothermia (TH) was not routinely used as a standard of care for asphyxiated neonates in our unit during the study period. The study group received magnesium sulphate intravenous infusion at $250 \mathrm{mg} / \mathrm{kg} / \mathrm{dose}$ (1 $\mathrm{ml} / \mathrm{kg} /$ dose in $20 \mathrm{ml}$ of $5 \%$ dextrose solution) over 1 hour within 6 hours of birth with 2 additional doses repeated after 24 hours and later at 48 hours. During the initial 72 hours of life all physiological variables were monitored continuously. Baseline serum magnesium was measured soon after delivery and two more serum magnesium levels were measured at 24 hours and at 48 hours in both the groups. Serum magnesium was measured using magnesium kit provided by CREST biosystems using Calmagite method. Blinding was not done due to settings limitation. 
The primary outcome of the present study included composite outcome of death or disability, developmental delay and neuromotor tone abnormality at 12 months follow up. The study evaluated developmental outcome at 12 months using Trivandrum Developmental Screening Chart. The Trivandrum Developmental Screening Chart was developed and designed by Child Development Center, SAT Hospital, Medical College, Trivandrum ${ }^{10}$. It contains 17 test items taken from norms given in Bayley Scale of Infant Development (Baroda norms) ${ }^{11}$. A vertical line is drawn at the level of chronological age of the infant being tested. If the infant fails to achieve any item that falls short on the left side of vertical line, the infant is considered to have developmental delay. Any obvious abnormality or asymmetry is also considered abnormal.

Amiel-Tisonneuromotor tone assessment was done by clinical evaluation of following: adductor angle, popliteal angle, foot dorsiflexion angle and scarf $\operatorname{sign}^{12,13}$. All statistical methods (descriptive statistics, chi square/contingency coefficient analysis, independent samples $t$ test) were carried out through the SPSS for windows (version 19.0). The $p$-value $<0.05$ was taken as statistically significant.

The study was approved by the Institutional Ethical Committee and informed consent was taken from parents.

\section{Results}

A total 142 newborns were found eligible for the study; of which 22 were excluded (14 had exclusion criteria, 5 parents refused to participate and 3 were discharged against medical advice before completion of intervention). Of remaining 120 newborns, 60 infants were randomly assigned to study group and 60 to control group. In magnesium group, 24 had mild HIE, 34 had moderate HIE and 2 had severe HIE. In placebo group, 27 had mild HIE, 32 had moderate HIE and 1 had severe HIE. HIE staging was done by Sarnat and Sarnat staging for Hypoxic ischemic encephalopathy 2 All baseline characteristic features, antenatal risk factors and severity of HIE were comparable between two groups. Baseline serum magnesium was measured soon after delivery and two more serum magnesium levels were measured at 24 hours and at 48 hours in both the groups. In infants of magnesium group, mean serum magnesium level increased from the baseline level of $1.52 \mathrm{meq} / \mathrm{l}$ to $2.72 \mathrm{meq} / \mathrm{l}$ at 48 hours. While in the control group mean serum magnesium level remained $1.62 \mathrm{meq} / \mathrm{L}$ at 48 hour. Based on the pharmacokinetics and estimates of plasma half life of magnesium sulphate as reported by Levene $M$ et al in 1995 , the neuroprotective range of serum magnesium is 2.4 - $5 \mathrm{meq} / \mathrm{l}$. In the present study, the post intervention mean serum magnesium level of study group was more than $2.4 \mathrm{meq} / \mathrm{l}(1.2 \mathrm{mmol} / \mathrm{l})$ which is in therapeutic and neuroprotective range. No adverse effects related to elevated levels of magnesium were noted in any of the cases. Normal neurobehaviour outcome was reported for 32 infants in study group (91.4\%) vs 22 infants in control group (68.75\%) among infants with moderate to severe HIE at discharge in our previous study $(p=0.019)$ which was statistically significant ${ }^{9}$.

Out of 120 infants, 69 infants had moderate to severe HIE during initial NICU stay. Total 41 infants (38 with moderate and 3 with severe HIE) could be followed up among those 69 infants with moderate-severe HIE. Out of 41 infants, 22 were in study group who received magnesium and remaining 19 in control group who received placebo (Figure 1). At 12 months, two infants in study group and three infants in control group were on antiepileptic drug for seizures. The mean occipitofrontal circumference was comparable between infants of two groups ( $46.5 \pm 0.45$ vs $46.2 \pm 0.55)$ with $p$-value 0.06 . The infant characteristics evaluated at 12 months were tabulated in Table 1.

Of 22 infants assigned to magnesium therapy for whom adequate data on the primary outcome were available,3(13.6\%) died or survived with neurodevelopmental disability as compared with five of ninteen infants $(26.3 \%)$ assigned to placebo for whom adequate data were available on the primary outcome (relative risk, $0.51 ; 95 \%$ confidence interval [Cl],0.141.88; $p=0.32$ ).

There was no statistically significant difference in composite outcome of death or disability at 12 months between two groups.

The developmental outcome was evaluated by paediatric resident doctors or consultants who were trained in using Trivandrum Developmental Screening Chart (TDSC) during infants OPD follow up visits. Though TDSC was a screening test, it was used as the method was simple, could be completed within five minutes and could be done by any trained health worker. Developmental delay was reported in 3 of 22 infants in study group vs 5 of 19 infants in control group (relative risk, 0.51; 95\% confidence interval [Cl],0.14$1.88 ; p=0.32$ ). Ameil- Tisonneuromotor tone assessment revealed tone abnormality in three of twenty two infants in study group vs five of 19 infants in control group (relative risk, 0.65; 95\% confidence interval [Cl],0.16$2.54 ; p=0.53)$. There was no statistically significant difference in developmental outcome and neuromotor tone assessment at 12 months between two study groups (Table-2). 
22 excluded

- 14 had exclusion criteria

- 5 parents refused to participate

- 3 were discharged against medical advice before completion of intervention

120 newborns were randomized into two groups

- 10 did not have adequate data for analysis at 12 months

- 20 were lost to follow up

- 8 had incomplete assessment.

Followed to a Corrected Age of 12 Months.

22 had data for analysis at 12 months
Placebo Group

$(n=60)$
8 did not have adequate data for analysis at 12 months

- 22 were lost to follow up

- 11 had incomplete assessment.

Table 1: Characteristics of study and control group infants evaluated at 12 months

\begin{tabular}{lccc}
\hline Characteristics & Magnesium group (n=22) & Placebo group(n=19) & $p$-value \\
\hline Female sex & $10(45.45 \%)$ & $11(57.89 \%)$ & 0.43 \\
\hline Birth weight: kg & $2.95 \pm 0.45$ & $3.10 \pm 0.56$ & 0.34 \\
\hline Gestational age (weeks) & $38.4 \pm 1.8$ & $38.6 \pm 1.5$ & 0.70 \\
\hline Moderate- severe HIE & $22(100 \%)$ & $19(100 \%)$ & 1.0 \\
\hline Age of surviving infants at follow-up (months) & & 12.7 & - \\
\hline Median & 12.4 & $12.4-13.7$ & - \\
\hline Interquartile range & $12.2-13.5$ & $3(15.8 \%)$ & 0.52 \\
\hline Seizure requiring antiepileptics & $2(9.09 \%)$ & $10.7 \pm 0.15$ & 0.12 \\
\hline Weight (kg) & $10.2 \pm 0.16$ & $74.5 \pm 0.54$ & 0.23 \\
\hline Length (cm) & $73.3 \pm 0.51$ & $46.2 \pm 0.55$ & 0.06 \\
\hline Head circumference $(\mathrm{cm})$ & $46.5 \pm 0.45$ & & \\
\hline
\end{tabular}


Table 2: Primary outcome of death or neurodevelopmental disability at 12 months

\begin{tabular}{lccccc}
\hline Outcome & $p$-value & 95\% confidence interval & Relative risk & Placebo & Magnesium \\
\hline Composite: Death or disability & 0.32 & $0.14-1.88$ & 0.51 & $5 / 19(26.3 \%)$ & $3 / 22(13.6)$ \\
\hline Developmental delay & 0.32 & $0.14-1.88$ & 0.51 & $5 / 19(26.3 \%)$ & $3 / 22(13.6 \%)$ \\
\hline Abnormal neuromotor tone & 0.53 & $0.16-2.54$ & 0.65 & $4 / 19(21.1 \%)$ & $3 / 22(13.6 \%)$ \\
\hline
\end{tabular}

\section{Discussion}

The baseline characteristics of both groups were comparable for birth weight, gestational age, sex and severity of HIE. All 41 infants with adequate data on the primary outcome had moderate-severe HIE during initial NICU stay. One infant in each group with severe HIE expired in the initial neonatal period.

In the study by Geeta Gathwala et al $^{14} 40$ term neonates with perinatal asphyxia were randomly assigned to magnesium therapy group and placebo group. A total 32 infants were available for follow up at 6 months which revealed abnormal or suspected developmental outcome in $18.75 \%$ vs $31.25 \%$ ( $p$-value $>0.05$ ) infants in magnesium and placebo group respectively. The developmental assessment was done with Denver 11. In the present study, the developmental assessment found no significant difference between study group and control group with a developmental delay of $13.6 \%$ vs $26.3 \%$ respectively in each group. $(p=0.32)$. Trivandrum Developmental Screening Chart was used in present study for developmental assessment which was easier and could be done at a community level.

Ichiba $\mathrm{H}$ et al in 2006 evaluated 30 infants with moderate to severe HIE who were given magnesium sulphate infusion in first 72 hours of life. Two infants died and remaining 28 infants were evaluated at 18 months. $21.6 \%$ of infants had severe neurodevelopmental disability at 18 months. The study was not a randomized controlled trial. No significant adverse effect was noticed $^{15}$.

In the present study, though more number of infants in study group had normal development compared to control group ( $86.4 \%$ vs 73.7 ), it did not reach a statistically significant level. Similar result was obtained in previously mentioned studies ${ }^{14,15}$.

Postnatal magnesium therapy in asphyxiated newborn was found to produce good short term neurological outcome in treatment group compared to control group in our own initial study ${ }^{9}$. Similar favorable short term outcome at discharge was noticed in other studies $^{16,17}$.
Magnesium was given in all studies in a dose of $250 \mathrm{mg} / \mathrm{kg}$ for initial 72 hours which ensured serum magnesium levels in the neuroprotective range which is 2.4-5 meq/l based on the pharmacokinetics and estimates of plasma half life as reported by Levene et $\mathrm{al}^{18}$. No short term or long term adverse effects were reported in any studies which emphasize that magnesium is an extremely safe drug when given under supervision.

There are many limitations in the present study. The study evaluated infants at 12 months and at this period motor development could be evaluated with reasonable accuracy. For cognitive and other socio-development evaluation, assessment at 18-24 months would be more accurate.

The study used Trivandrum Development Screening Chart (TDSC) for assessing developmental delay which is not a confirmatory method of developmental assessment.TDSC was designed by selecting 17 test items from BSID (Baroda Norms). It was validated both at hospital and community level against DDST. The 17 items contain different domains of development till two years of age. A vertical line is drawn at the level of age of child in months being tested. If child fails to achieve any item that falls short on the left side of vertical line the child is considered to have developmental delay. It was used as the method was simple, could be completed within five minutes and could be done by any trained health worker with more community implications ${ }^{10}$.

The attrition rates for both groups were high in the study. The final assessment could only be done for $37 \%(22 / 60)$ in magnesium group and $32 \%(19 / 60)$ in placebo group. The follow up is very poor in majority of our government tertiary hospitals as patients are coming from far distant remote villages and were poor in socioeconomic and educational background. So sample size was small. This has been a limitation of the study. Still we managed to follow up 41 out of 69 infants with moderate-severe $\mathrm{HIE}(59 \%)$.

In addition to clinical neurological examination, neuroimaging and EEG might give more information regarding neurological status of surviving infants with 
perinatal asphyxia ${ }^{19,20}$. Though cranial ultrasound was done during NICU stay for 35 of 41 follow up infants, we could not evaluate the infants with EEG or MRI. Cranial ultrasound was normal for 32 out of 35 infants. Out of three infants with abnormal cranial USG, two had intraventricular haemorrhage and one had periventricular leukomalacia. This again emphasize that cranial USG has a very low sensitivity for detecting abnormalities for infants with HIE. MRI and EEG was not done due to resource limitations in our set-up.

During the study period, our unit had not started therapeutic hypothermia $(\mathrm{TH})$ routinely as a standard of care for all asphyxiated neonates. The low resource setting and lack of experience/expertise with this modality were some of the reasons behind it. The guidelines of $\mathrm{WHO}$ collaborating centre for training and research in newborn care, All India institute of medical science mentions that: "TH has become standard of care in developed countries. However, in low to middle income countries where the patient profile is different (concomitant IUGR, infection and nutritional deficiencies), and there is a paucity of intensive care, and many births occur out of hospital, small studies have shown that there may be increase in mortality with $\mathrm{TH}$. The true value of $\mathrm{TH}$ in low to middle income countries is yet to be tested."

So we intended to do the study with magnesium sulphate which is a low cost drug with high margin of safety, hence suitable for a resource limited settings. In addition to that there is very little literature regarding long term outcome with magnesium therapy.

The favourable short term outcome related to magnesium might be probably due to following properties of magnesium-its central anticonvulsant effect on hippocampal seizures, cerebral vasodilatory properties, reduction of calcium influx by gating NMDA receptor in brain and antagonizing glutamate excitotoxicity. The neurodevelopmental outcome was no longer significant at 12 months age. Development was assessed by a screening method only. In addition, low sample size due to high attrition rate and poor follow up might have also affected this disparity in outcome. Hence, further adequately powered research is needed to evaluate the effect of magnesium in asphyxia.

\section{Conclusion}

The available data states that magnesium as a novel agent may result in favourable short term neurological outcome. But long term follow up studies are sparse in literature. Our study found that there was no difference in the composite outcome of death or disability at 12 months when postnatal magnesium therapy was given as a neuroprotective strategy for perinatal asphyxia.The present evidence does not prove that magnesium therapy results in a favourable long term neurodevelopmental outcome, though no significant adverse effect has been documented.

\section{References}

1. World Health Organization. Maternal and Newborn Health/Safe Motherhood Unit, Division of Reproductive Health. Basic Newborn Resuscitation: A Practical Guide. Geneva: World Health Organization 1999;1018.

2. Sarnat HB, Samat MS. Neonatal encephalopathy following fetal distress: A clinical and electroencephalographic study. Arch Neurol 1976:33: 696-705.

3. Volpe JJ. Perinatal brain injury: From pathogenesis to neuroprotection. Ment Retard Dev Disabil Res Rev 2001;7(1):56-64.

4. Garnier Y, Jensen A. Perinatal brain damagefrom pathophysiology to prevention. Eur J ObstetGynecolReprodBio/2003;110:S70-S79.

5. Vannucci RC. Current and potentially new management strategies for perinatal hypoxic-ischemic encephalopathy. Pediatrics 1990;85;961-68.
6. Choi DW. The role of glutamate neurotoxicity in hypoxic-ischemic neuronal death. Annu Rev Neurosci 1990;13:171-82.

7. Haddad JJ. N-methyl-D-aspartate (NMDA) and the regulation of mitogen-activated protein kinase (MAPK) signaling pathways: A revolving neurochemical axis for therapeutic intervention? ProgNeurobio/2005;77:252-82.

8. Chahal H, D'Souza SW, Barson AJ, Slater P. Modulation by magnesium of $\mathrm{N}$-methyl-D-aspartate receptors in developing human brain. Arch Dis Child Fetal Neonatal Ed 1998; 78(2):F116-F120.

9. Savitha MR, Rajprakash. Beneficial effect of intravenous magnesium sulphate in term neonates with perinatal asphyxia. Int $J$ ContempPediatr 2016;3(1):150-54.

10. Nair MK, George B, Philip E, Lekshmi MA, Haran JC, Sathy N. Trivandrum developmental screening chart. Indian Pediatr1991;28(8):869-72. 
11. Phatak P, DhapreM,PanditAN,Kulkarni S. A study of Baroda developmental screening test for infants. Indian Pediatr1991;28:843-49.

12. Amiel-Tison C. Update of the Amiel-Tison neurologic assessment for the term neonate or at 40 weeks corrected age. PediatrNeurol2002;27(3):196-212.

13. Chaudhari $S$ and Deo B.Neurodevelopmental Assessment in the First Year with Emphasis on Evolution of Tone. Indian Pediatr2006;43:527-34.

14. Gathwala G, Khera A, Singh J, Balhara B. Magnesium for neuroprotection in birth asphyxia. J PediatrNeurosci 2010;5(2):102-4.

15. Ichiba H, Yokoi T, Tamai H, Ueda T, Kim T, Yamano T. Neurodevelopmental outcome of infants with birth asphyxia treated with magnesium sulfate. PediatrInt. 2006;48(1):70-5.

16. Ichiba H, Tamai H, Negishi H, Ueda T, Kim TJ, Sumida $Y$, et al. Randomized controlled trial of magnesium sulfate infusion for severe birth asphyxia. Pediatrlnt2002;44(5):505-9.

17. Bhat MA, Charoo BA, Bhat JI, Mushtaq S, Ali SW, Mufti $\mathrm{MH}$. Magnesium Sulfate in severe perinatal asphyxia: A randomized placebo-controlled trial. Pediatrics2009;123(5);e764-e769.

18. Levene M, Blennow M, Whitelaw A, Hanko E, Fellman V, Hartley R. Acute effects of two different doses of magnesium sulphate with birth asphyxia. Arch Dis Child 1995;73(3): F174-F177.

19. Murray DM, Boylan GB, Ryan CA, Connolly S. Early EEG Findings in Hypoxic-Ischemic Encephalopathy Predict Outcomes at 2 Years. Pediatrics 2009;124(3):e459-67. doi: 10.1542/peds.2008-190.

20. Biagioni E, Mercuri E, Rutherford M, Cowan F, Azzopardi D, Frisone MF, Cioni G, Dubowitz L. Combined use of electroencephalogram and magnetic resonance imaging in full-term neonates with acute encephalopathy. Pediatrics 2001;107(3):461-68. 